Philosophie ANTIQUE

\section{Philosophie antique}

Problèmes, Renaissances, Usages

$10 \mid 2010$

Philosophie et mathématiques

\title{
Maria Michela SASSI, Gli inizi della filosofia : in Grecia
}

Turin, Bollati Boringhieri, 2009, 307 p., ISBN 978-88-339-1974-4, € 19,00.

Leopoldo Iribarren

\section{OpenEdition}

Journals

Édition électronique

URL : https://journals.openedition.org/philosant/2352

DOI : 10.4000/philosant.2352

ISSN : 2648-2789

Éditeur

Éditions Vrin

\section{Édition imprimée}

Date de publication : 30 octobre 2010

Pagination : 267-271

ISBN : 978-2-7574-0179-8

ISSN : 1634-4561

\section{Référence électronique}

Leopoldo Iribarren, « Maria Michela sassı, Gli inizi della filosofia : in Grecia », Philosophie antique [En ligne], 10 | 2010, mis en ligne le 11 juillet 2019, consulté le 05 décembre 2022. URL : http://

journals.openedition.org/philosant/2352 ; DOI : https://doi.org/10.4000/philosant.2352

\section{(c) $\oplus \Theta \Theta$}

Creative Commons - Attribution - Pas d'Utilisation Commerciale - Pas de Modification 4.0 International - CC BY-NC-ND 4.0

https://creativecommons.org/licenses/by-nc-nd/4.0/ 


\section{COMPTES RENDUS}

Maria Michela SASSI, Gli iniz̨i della filosofia : in Grecia, Turin, Bollati Boringhieri, 2009, 307 p., ISBN 978-88-339-1974-4, € 19,00.

Le regain d'intérêt de ces derniers temps pour la philosophie présocratique est avant tout perceptible dans le nombre d'ouvrages collectifs importants dont cette période de l'histoire de la pensée fait l'objet depuis une dizaine d'années. Les divers "companions », "handbooks » et autres collections d'études qui lui sont consacrées, sans oublier les actes des Symposia Praesocratica, qui visent l'exploration suivie de ce domaine, donnent une idée de la complexification croissante des problématiques. Moins nombreux sont cependant les ouvrages d'auteurs individuels qui, dans une approche innovante, offrent une vision d'ensemble de la période, comme c'est le cas du livre de M.M. Sassi. Il faut dire d'emblée que cet ouvrage n'est pas une histoire philosophique des doctrines. L'auteur défend en revanche une approche multidimensionnelle (c'est le sens du pluriel du titre) des origines de la philosophie grecque, susceptible de tenir compte du foisonnement de ses objets de recherche et de la diversité de ses démarches et de ses régimes discursifs. Cette approche s'accommode bien de l'indétermination des frontières d'une discipline dont l'unité et l'autonomie sont pour le moins problématiques. Pour ce qui est de la méthode, M.M. Sassi se réclame d'une forme d'historiographie "prospective» (dans le sens que M. Frede donnait à cette expression), c'est-à-dire d'une tentative d'expliciter les intentions intellectuelles des acteurs, qui savent d'où ils partent et où ils veulent aller, mais ne peuvent pas prévoir les possibles obstacles et les détours qu'ils trouveront sur leur chemin (p. 15 sq.). Le résultat est un remarquable exemple d'histoire intellectuelle qui offre une description riche et complexe - avec une tendance cependant à privilégier une approche sociologique des formes discursives archaïques - de ce que les premiers philosophes furent en mesure d'accomplir, et de leur interaction avec le reste de la société.

Le premier chapitre, "Talete padre della filosofia ? », revient sur la problématique liée à la construction historiographique aristotélicienne qui fait de Thalès le père de la philosophie. L'analyse est particulièrement éclairante en ce qu'elle montre comment la détermination de l'origine positive d'une pratique savante est toujours déjà investie par un certain intérêt de la connaissance, voire par une idéologie. Le cas de Thalès est à cet égard paradigmatique, car son propos fondateur sur l'eau a pu être interprété par les modernes tantôt comme la première expression d'une curiosité rationnelle pour les phénomènes physiques (Burnet, Gomperz, etc.), tantôt comme un postulat ontologique aux accents mystiques (Nietzsche, Heidegger, Colli). M.M. Sassi consacre aussi une partie du chapitre à l'examen de cette autre tradition historiographique qui situe les origines de la philosophie au Moyen-Orient. S'il est vrai qu'on trouve cette opinion 
chez Hérodote et Platon, ce qui intéresse surtout l'auteur est son développement moderne, notamment au sein de l'école « ritualiste» de Cambridge. Les pages qu'elle consacre spécifiquement à l'œuvre de F.M. Cornford mettent en lumière les mécanismes internes d'une approche qui vise à supprimer ce que J.-P. Vernant qualifiera plus tard de "mutation» entre anciennes et nouvelles formes de pensée. L'ouverture «anticlassiciste» de Cornford est évaluée favorablement par l'auteur dans la mesure où elle eut le mérite de diriger l'attention vers un matériau oriental qui est certainement présent dans les cosmologies grecques. Cependant, M.M. Sassi remarque pertinemment que "l'obsession de continuité » (p. 47) chez Cornford aboutit à un rejet total d'un certain apport irréductible qui fait que les cosmologies grecques sont à l'origine de développements intellectuels qui n'ont pas de contrepartie au Moyen-Orient. La position de l'auteur dans ce débat est intéressante car elle cherche à dépasser l'opposition traditionnelle mythos/logos tout en restant attachée à la lettre du passage de la Métaphysique (A, 3, 983b-984a2) d'Aristote où cette opposition est, d'une certaine manière, exprimée pour la première fois. M.M. Sassi montre en effet qu'Aristote trace dans ce passage difficile les lignes de continuité et de rupture, tant au niveau du contenu que de la forme, entre les représentations du mythe et le propos inédit de Thalès. Pour M.M. Sassi, la clarté expressive qu'Aristote attribue à Thalès constitue, avec la tendance à éliminer le recours à l'intervention divine dans la nature, et une certaine dose d'empirisme, une des marques les plus nettes de l'émergence de la philosophie. Toutefois, comme le fait remarquer l'auteur, il reste difficile de distinguer à l'époque archaïque une démarche proprement philosophique des autres pratiques savantes, elles-mêmes en voie de différenciation (médecine, astronomie, mathématique, ou encore sagesse ou simple curiosité à l'égard du monde). La philosophie, par l'indétermination même de son objet, constitué par "la totalité des choses », est plus exposée que les autres disciplines aux refigurations constantes.

Le deuxième chapitre, "Filosofia nelle cosmogonie", renverse en quelque sorte la problématique développée dans le premier : il s'agit ici d'identifier les éléments philosophiques que l'on trouve dans les formes discursives traditionnelles à l'époque archaïque. Dans la lignée de H. Fränkel et de H. Diller, M.M. Sassi défend l'idée que la Théogonie d'Hésiode porte déjà les marques d'une pensée systématique et d'une forme de spéculation philosophique. Pour étayer son affirmation, elle déploie une comparaison avec la cosmogonie d'Anaximandre, notamment en ce qui concerne la nature des principes des deux systèmes, à savoir l'apeiron et Chaos. Si la comparaison est éclairante à certains égards (en particulier à propos des connotations politiques liées à la fonction des principes), on peut cependant objecter à l'interprétation de M.M. Sassi une tendance à assimiler Chaos et apeiron qui fait d'Hésiode le tenant d'un principe unique (p. 70 sqq.). Il nous semble, au contraire, qu'Hésiode laisse la possibilité ouverte à deux types de principe, de différence et d'identité, pour produire la cosmogonie selon le schéma généalogique, même si parmi ces principes c'est effectivement Chaos qui est né en premier. En somme, Hésiode semble faire preuve ici d'une forme d'«approche multiple» d'un même problème, un mode de raisonnement que M.M. Sassi analyse ailleurs comme étant caractéristique de 
la forme mythique, par opposition aux modes mobilisés par les philosophes ioniens (p. 100 sqq.). Quelques pages sont consacrées dans ce même chapitre à la cosmogonie de Phérécyde, entendue comme un projet de rationalisation du patrimoine mythologique, et aussi au statut «mixte » (à la fois «théologien » et philosophe) qu'Aristote accorde à son auteur. Mais peut-être l'analyse la plus fine de cette partie concerne-t-elle le célèbre fragment "cosmologique » d'Alcman, qui divise les commentateurs entre ceux qui le considèrent comme précurseur des cosmologies philosophiques (H. Fränkel, Kirk-Raven-Schofield) et ceux qui pensent que le genre lyrique auquel il appartient exclut d'emblée ce type de spéculation (G. Most). La position de M.M. Sassi consiste à avancer l'idée qu'une forme de "cosmogonie soft» (p. 98 sq.), selon laquelle un poète archaïque, prenant une distance par rapport à la tradition existante, fait intervenir des divinités auxquelles il attribue des pouvoirs relatifs à la production ou au maintien de l'ordre cosmique, n'est pas en contradiction avec l'unité générique de l'œuvre. Cette notion de «cosmogonie soft» mériterait d'être approfondie, car il semblerait qu'elle soit de fait l'une des voies privilégiées par les poètes archaïques pour pratiquer la spéculation cosmologique sans pour autant cesser de se conformer aux attentes d'un type spécifique de composition.

Le troisième chapitre, intitulé «Prove di scrittura », est consacré à la vaste question de la rationalisation entendue comme condition formelle de l'émergence de la philosophie. Tout en admettant qu'il est difficile d'établir une causalité directe entre la structure sociale de la polis et la rationalité spécifiquement philosophique, M.M. Sassi considère, comme J.-P. Vernant ou G. Lloyd, que la cité grecque n'en offre pas moins un cadre argumentatif et agonistique favorable à l'émergence de nouvelles formes de pensée. Or si l'auteur prend en considération dans son analyse d'autres facteurs positifs déterminants, comme la religion ou l'expansion coloniale, c'est surtout la publicité de la forme, notamment la diffusion de l'écriture, qui retient son attention. Dans ce chapitre, M.M. Sassi fait un usage pertinent de la notion de "mémoire culturelle » élaborée par J. Assmann pour décrire le rapport des premiers philosophes à l'écrit, en particulier chez Anaximandre, Xénophane et Héraclite. On retiendra comme la plus novatrice la lecture d'Anaximandre, où l'auteur prolonge une réflexion amorcée dans un article important ( Anassimandro e la scrittura della 'legge' cosmica », dans Sassi (éd.), La costružione del discorso filosofico nell'età dei Presocratici, Pise, 2006, p. 3-26) consacré à la question de l'institution de la prose philosophique comme modalité discursive à la recherche d'une légitimité dont ne jouissait à l'époque archaïque que la parole autorisée du poète. Une convaincante analyse sémantique des termes présents dans le fragment B1 montre comment l'écriture de la loi cosmique puise son autorité dans un modèle juridique dont les traits les plus marquants sont une tendance à la formulation impersonnelle et concise et l'inscription de sa validité générale dans le temps. Contrairement à une tendance, courante depuis les travaux de G. Vlastos et de J.-P. Vernant, à interpréter le fragment en se focalisant sur l'idée d'équilibre entre les opposés, M.M. Sassi propose une lecture qui, à la lumière de l'analogie entre réalité physique et réalité politique, privilégie plutôt le point de vue du procès et de la conflictualité à l'intérieur du cosmos. Cette lecture mobilise par ailleurs des rapprochements 
thématiques et historiques avec Solon et Héraclite qui sont d'un grand intérêt non seulement pour l'élucidation des doctrines physiques proprement dites, mais aussi pour ce qui concerne la problématique de leurs conditions d'énonciation.

La question de l'âme et de son destin est abordée par M.M. Sassi dans le quatrième chapitre, intitulé «Vicende dell'anima ». L'originalité de son approche consiste à intégrer dans un même ensemble la problématique de la théorie de la connaissance chez les philosophes présocratiques avec celle de la formation des diverses conceptions de l'âme, voire, selon les cas, avec celle des doctrines eschatologiques. Cette approche conjointe permet de saisir les premières manifestations d'un problème philosophique qui trouve sa formulation la plus aboutie dans le Timée de Platon. Pour son analyse, l'auteur emprunte à G. Betegh un schéma fort efficace selon lequel on distingue dans l'architectonique des systèmes présocratiques deux types idéaux du rapport âme/cosmos. Il y a d'un côté un "journey model» (les pythagoriciens, les orphiques) où l'âme de l'individu, traitée comme une entité atomique, garde son identité et sa mémoire tout au long d'un parcours à travers des régions valorisées axiologiquement, donnant lieu à une pensée de l'individu, voire à une eschatologie de la rétribution. De l'autre, il y a le "portion model » (Anaximène, Héraclite) selon lequel l'âme est faite d'un ou de plusieurs éléments constitutifs de l'univers et interagit avec lui, de telle manière que la matière responsable des fonctions psychiques tient aussi un rôle cosmologique ; dans cette perspective, l'équivalent du sujet qui pense doit être trouvé dans l'objet qui est une totalité. Dans ce schéma, Empédocle mérite une place à part, car, comme le signale M.M. Sassi, il constitue une tentative de penser ensemble les deux modèles, même si le daimon empédocléen n'est pas assimilable à ce qu'il entendait par psyche (p. 188 sqq.). De manière plus générale, la question touche au sens que l'on donne à la coexistence des deux récits d'Empédocle, ce qui implique l'élucidation du type de rapport qui relie les deux cycles, le cosmique et le démonique. M.M. Sassi ne prétend pas résoudre cette question, certainement l'une des plus discutées de l'histoire de la philosophie archaïque, elle a cependant le mérite de la développer dans toute sa complexité.

Enfin, le cinquième et dernier chapitre, intitulé «Voci autorevoli », concerne la problématisation par les penseurs archaïques de ce nouvel objet de connaissance que constitue la nature. Si les Milésiens identifient et fixent cet objet dans la prose, dont l'énonciation et les prétentions à la vérité sont inconditionnées, ce sont Xénophane et Héraclite qui, dans des formes qui favorisent le métadiscours, élaborent les premières réflexions épistémologiques. On voit apparaitre chez ces auteurs, mais aussi, dans une certaine mesure, chez Alcméon et Hécatée, de véritables théorisations de la connaissance humaine, dont les aspects les plus saillants qui ressortent de l'analyse de M.M. Sassi sont, d'une part, la thématisation de ses limites, et, de l'autre, celle de la dimension pragmatique et historique (le discours des mortels vaut dans la mesure où il est accepté par autrui, non comme une révélation, mais à titre de construction provisoire). Dans ce contexte, que l'on pourrait qualifier de laïque, l'auteur s'interroge sur le retour à un savoir révélé chez Parménide et Empédocle. Aux yeux de M.M. Sassi, l'hexamètre s'impose à ces auteurs comme la forme la plus adaptée à la solennité 
d'un contenu particulièrement élevé (p. 219 sqq.). Plutôt que sur les doctrines correspondant aux deux parties du poème, sa lecture de Parménide est axée sur la katabasis qui est figurée dans le proème, où elle reconnait une expérience de type mystique qui contraint l'auteur à l'adoption de l'hexamètre. Dans le cas d'Empédocle, c'est aussi l'expérience religieuse qui est mobilisée par M.M. Sassi pour expliquer le recours à l'hexamètre. Son interprétation opère un retour, avec quelques aménagements, au schéma biographique selon lequel les Catharmes succèdent au poème physique et sont le fruit d'une conversion religieuse. Les développements sur l'autoreprésentation d'Empédocle dans ce poème sont cependant innovants.

Grâce à l'intelligence et à l'originalité de son approche, la succession des philosophes présocratiques apparait sous un éclairage nouveau dans ce livre. Destiné à un public élargi, il n'en demeurera pas moins une référence incontournable pour le travail plus spécialisé.

Leopoldo IRIBARREN

Adam DrozdeK, In the Beginning was the Apeiron: Infinity in Greek Philosophy, Stuttgart, Franz Steiner Verlag, 2008 (Klassische Philologie, Palingenesia, 94), 173 p. ISBN 978-3-515-09258-6.

Cette monographie présente une étude de l'infini dans la philosophie grecque, d'Anaximandre à l'ancien stö̈cisme. Elle comporte une table des matières, une préface de deux pages, seize chapitres organisés de façon chronologique, un index des noms propres, et un index des sources anciennes. Il n'y a pas de bibliographie (les références aux auteurs modernes sont données en notes). Cinq chapitres sont des reprises (modifiées le cas échéant) d'articles publiés dans Hermes, Diálogos et Eos ${ }^{1}$.

Disons-le d'emblée : cet ouvrage est décevant. Étant donné le nombre de critiques dont il peut faire l'objet, nous n'en présenterons pas le contenu chapitre par chapitre. La déception saisit le lecteur dès la préface. La thèse de Drozdek (D.) est énoncée ainsi : "Greek intellectual history clearly shows that the Greeks had none of the horror infiniti that sometimes is ascribed to them » (p. 8). Cependant, D. ne prend pas la peine de mettre en évidence l'originalité de son travail par rapport aux études existantes ${ }^{2}$. En des termes très généraux, voire généralisateurs, il se contente de mentionner l'omniprésence de l'infini dans la philosophie grecque en faisant une liste de "problèmes" (" the problem of the infinite size of the

1. L'auteur remercie les éditeurs de ces revues à la fin de la préface (p. 8), mais ne donne nulle part la référence exacte de chaque article.

2. Les livres de T.G. Sinnige (Matter and Infinity in the Presocratic Schools and Plato, 1971) et de L.S.J. Sweeney (Infinity in Presocratics: a Bibliographical and Philosophical Study, 1972) sont cités en notes pour des points particuliers dans le corps de l'ouvrage. Curieusement, nulle mention n'est faite de l'ouvrage de R. Mondolfo, L'Infinito nel pensiero dell'antichità classica, 1956. 